\title{
Increased Serum Malondialdehyde-Modified Low-Density Lipoprotein and Coronary Angiographic Progression After Drug-Eluting Stent Implantation in Patients With Stable Angina
}

\author{
Masashi Yokoi, MD; Tsuyoshi Ito, MD; Hiroshi Fujita, MD; \\ Tomonori Sugiura, MD; Yoshihiro Seo, MD; Nobuyuki Ohte, MD
}

\begin{abstract}
Background: Cardiac events can occur after drug-eluting stent (DES) implantation due to coronary plaque progression at nonstented sites. Malondialdehyde-modified low-density lipoprotein (MDA-LDL) is suggested to be an atherogenic marker. This study investigated the relationship between serum MDA-LDL and angiographic progression after DES implantation.

Methods and Results: In total, 207 patients who underwent percutaneous coronary intervention (PCI) using DES and follow-up coronary angiography were retrospectively analyzed. MDA-LDL was serially measured before $\mathrm{PCl}$ and at follow up. Persistent high MDA-LDL was defined as a MDA-LDL level more than the median value both before $\mathrm{PCl}$ and at follow up. Angiographic progression was assessed by serial analysis of quantitative coronary angiography. Angiographic progression occurred in 35 patients (16.9\%). MDA-LDL before $\mathrm{PCl}$ was significantly higher in the progression group than the non-progression group in all patients (143.4 $\pm 35.8 \mathrm{U} / \mathrm{L}$ vs. $103.0 \pm 33.5 \mathrm{U} / \mathrm{L}, \mathrm{P}<0.001)$ and in patients with controlled LDL-cholesterol (LDL-C $<100 \mathrm{mg} / \mathrm{dL}$ both before $\mathrm{PCl}$ and at follow up; 121.8 $\pm 32.7 \mathrm{U} / \mathrm{L}$ vs. $84.9 \pm 24.9 \mathrm{U} / \mathrm{L}, \mathrm{P}<0.001)$. There were positive correlations between $\%$ diameter stenosis changes and serum MDA-LDL before $\mathrm{PCl}$ in all patients $(r=0.33, \mathrm{P}<0.01)$ and those with controlled LDL-C $(r=0.23, \mathrm{P}=0.04)$. In multivariate logistic regression analysis, persistent high MDA-LDL was an independent predictor of plaque progression.
\end{abstract}

Conclusions: Increased serum MDA-LDL was associated with angiographic progression after DES implantation.

Key Words: Angiographic progression; Coronary artery disease; Malondialdehyde-modified low-density lipoprotein; Quantitative coronary angiography

$\mathbf{T}$ he appearance and development of drug-eluting stents (DES) have markedly reduced adverse cardiac events, including target lesion revascularization (TLR) after percutaneous coronary intervention (PCI).1,2 In addition, lipid-lowering pharmacological therapy has been established as a cornerstone of reducing the risk of coronary artery disease (CAD) ${ }^{3,4}$ However, cardiac events can occur even under adequate lipid-lowering therapy after PCI with DES, due to the development of new lesions at non-stented segments. ${ }^{5}$ Therefore, it is clinically important to predict and prevent CAD progression, as it is a strong predictor of future cardiac events. ${ }^{6}$ Although complex plaque morphology, ${ }^{7}$ enhanced inflammation, ${ }^{8}$ and impaired lipid profiles 9 may be responsible for plaque progression, the underlying mechanisms are not fully elucidated.

Oxidative modification of low-density lipoprotein (LDL) plays a key role in the formation and acceleration of atherosclerosis. ${ }^{10}$ Malondialdehyde-modified LDL
(MDA-LDL), which is an epitope of oxidized LDL, was recently shown to be a marker of CAD severity ${ }^{11}$ and plaque vulnerability, ${ }^{12}$ as well as a predictor of acute coronary syndrome ${ }^{13}$ and adverse cardiac events after PCI using DES. ${ }^{14}$ However, it remains unclear whether abnormal serum MDA-LDL level contributes to angiographic plaque progression of non-PCI lesions.

The aim of this study was to clarify the relationship between serial serum MDA-LDL levels and angiographic progression at non-PCI target lesions in patients with stable angina undergoing lipid-lowering therapy after DES implantation.

\section{Study Population}

From June 2009 to January 2014, a total of 604 patients with stable angina underwent PCI at our institute. Of

Received January 28, 2020; revised manuscript received June 17, 2020; accepted June 29, 2020; J-STAGE Advance Publication released online August 28, 2020 Time for primary review: 34 days

Department of Cardiology, Nagoya City University Graduate School of Medical Sciences, Nagoya, Japan

Mailing address: Tsuyoshi Ito, MD, Department of Cardiology, Nagoya City University Graduate School of Medical Sciences, 1 Kawasumi, Mizuhocho, Nagoya 467-8601, Japan. E-mail: tuyosiito@gmail.com

All rights are reserved to the Japanese Circulation Society. For permissions, please e-mail: cj@j-circ.or.jp

ISSN-1346-9843 


\begin{tabular}{|c|c|c|c|c|}
\hline Variables & $\begin{array}{c}\text { All } \\
(n=207)\end{array}$ & $\begin{array}{l}\text { Progression } \\
(n=35)\end{array}$ & $\begin{array}{c}\text { Non-progression } \\
(n=172)\end{array}$ & $P$ value \\
\hline Age (years) & $71.1 \pm 8.4$ & $68.7 \pm 9.0$ & $71.5 \pm 8.3$ & 0.07 \\
\hline Male & $148(71.5)$ & $27(77.1)$ & $121(70.3)$ & 0.42 \\
\hline $\mathrm{BMI}\left(\mathrm{kg} / \mathrm{m}^{2}\right)$ & $24.2 \pm 3.3$ & $24.5 \pm 2.5$ & $24.1 \pm 3.4$ & 0.43 \\
\hline HT & $184(88.9)$ & $32(91.4)$ & $152(88.4)$ & 0.77 \\
\hline DM & $89(43.0)$ & $17(48.6)$ & 72 (41.9) & 0.47 \\
\hline Smoking & $54(26.1)$ & $9(25.7)$ & 45 (26.2) & 0.96 \\
\hline Previous $\mathrm{PCl}$ & $73(35.3)$ & $16(45.7)$ & $57(33.1)$ & 0.16 \\
\hline Previous CABG & $13(6.3)$ & $1(2.9)$ & $12(7.0)$ & 0.70 \\
\hline MVD & $89(43.0)$ & $20(57.1)$ & $69(40.1)$ & 0.06 \\
\hline CKD & $66(31.9)$ & $8(22.9)$ & $58(33.7)$ & 0.21 \\
\hline History of MI & $68(32.9)$ & $13(37.1)$ & $55(32.0)$ & 0.55 \\
\hline In-stent restenosis at follow up & $31(14.9)$ & $9(25.7)$ & $22(12.8)$ & 0.05 \\
\hline ID-TLR at follow up & $9(4.3)$ & $2(5.7)$ & $7(4.1)$ & 0.65 \\
\hline Follow-up period (months) & $10.3 \pm 2.3$ & $10.5 \pm 2.7$ & $10.2 \pm 2.2$ & 0.44 \\
\hline \multicolumn{5}{|l|}{ Reason for follow up } \\
\hline Onset of ischemic symptom & $22(10.6)$ & $5(14.3)$ & $17(9.9)$ & \\
\hline Objective signs of ischemia & $4(1.9)$ & $0(0)$ & $4(2.3)$ & \\
\hline Follow-up examination & $181(87.4)$ & $30(85.7)$ & $151(87.8)$ & 0.51 \\
\hline \multicolumn{5}{|c|}{ Non-target lesion maximum stenosis } \\
\hline Mild (0-25\%) & $21(10.1)$ & $1(2.9)$ & $20(11.6)$ & \\
\hline Moderate (26-75\%) & $109(52.7)$ & $19(54.3)$ & $90(52.3)$ & \\
\hline Severe $(76-100 \%)$ & $77(37.2)$ & $15(42.9)$ & $62(36.0)$ & 0.27 \\
\hline $\mathrm{SBP}$ at $\mathrm{PCl}(\mathrm{mmHg})$ & $136.5 \pm 18.6$ & $135.2 \pm 17.9$ & $136.8 \pm 18.8$ & 0.65 \\
\hline $\mathrm{DBP}$ at $\mathrm{PCl}(\mathrm{mmHg})$ & $73.1 \pm 10.7$ & $72.5 \pm 11.9$ & $73.3 \pm 10.5$ & 0.71 \\
\hline SBP at follow up $(\mathrm{mmHg})$ & $136.1 \pm 16.9$ & $131.6 \pm 13.8$ & $137.1 \pm 17.3$ & 0.08 \\
\hline DBP at follow up (mmHg) & $66.7 \pm 10.8$ & $65.5 \pm 9.2$ & $67.0 \pm 11.1$ & 0.46 \\
\hline \multicolumn{5}{|l|}{ Medications at $\mathrm{PCl}$} \\
\hline Statin & $133(64.3)$ & $25(71.4)$ & $108(62.8)$ & 0.33 \\
\hline Antihypertensive agents & $168(81.2)$ & $29(82.9)$ & $139(80.8)$ & 0.78 \\
\hline \multicolumn{5}{|l|}{ Antidiabetic agents } \\
\hline None & $134(64.7)$ & $22(62.9)$ & $112(65.2)$ & \\
\hline Oral & $60(29.0)$ & $8(22.9)$ & $52(30.2)$ & \\
\hline Insulin & $13(6.3)$ & $5(14.3)$ & $8(4.7)$ & 0.09 \\
\hline \multicolumn{5}{|l|}{ Medications at follow up } \\
\hline Statin & $194(93.7)$ & $34(97.1)$ & $160(93.0)$ & 0.70 \\
\hline Antihypertensive agents & $182(87.9)$ & $31(88.6)$ & $151(87.8)$ & 0.90 \\
\hline \multicolumn{5}{|l|}{ Antidiabetic agents } \\
\hline None & $130(62.8)$ & $21(60)$ & $109(63.4)$ & \\
\hline Oral & $63(30.4)$ & $9(25.7)$ & $54(31.4)$ & \\
\hline Insulin & $14(6.8)$ & $5(14.3)$ & $9(5.2)$ & 0.14 \\
\hline \multicolumn{5}{|l|}{ Laboratory data at $\mathrm{PCl}$} \\
\hline MDA-LDL (U/L) & $109.9 \pm 37.1$ & $143.4 \pm 35.8$ & $103.0 \pm 33.5$ & $<0.01$ \\
\hline LDL-C (mg/dL) & $102.7 \pm 29.1$ & $112.5 \pm 29.8$ & $100.8 \pm 28.7$ & 0.03 \\
\hline $\mathrm{HDL}-\mathrm{C}(\mathrm{mg} / \mathrm{dL})$ & $47.8 \pm 13.7$ & $44.2 \pm 12.1$ & $48.5 \pm 13.9$ & 0.09 \\
\hline TGs (mg/dL) & $146.4 \pm 64.3$ & $155.2 \pm 63.8$ & $144.6 \pm 64.4$ & 0.38 \\
\hline CRP (mg/dL) & $0.09(0.05-0.19)$ & $0.13(0.05-0.23)$ & $0.09(0.05-0.18)$ & 0.27 \\
\hline $\mathrm{HbA1c}(\%)$ & $6.34 \pm 0.95$ & $6.58 \pm 1.02$ & $6.30 \pm 0.93$ & 0.11 \\
\hline \multicolumn{5}{|l|}{ Laboratory data at follow up } \\
\hline MDA-LDL (U/L) & $105.8 \pm 32.5$ & $134.5 \pm 36.0$ & $100.0 \pm 28.4$ & $<0.01$ \\
\hline LDL-C (mg/dL) & $95.0 \pm 26.7$ & $106.0 \pm 31.6$ & $92.8 \pm 25.1$ & $<0.01$ \\
\hline HDL-C (mg/dL) & $49.8 \pm 13.3$ & $45.5 \pm 11.1$ & $50.6 \pm 13.5$ & 0.04 \\
\hline TGs (mg/dL) & $151.5 \pm 73.4$ & $174.3 \pm 77.5$ & $146.8 \pm 71.9$ & 0.04 \\
\hline CRP (mg/dL) & $0.08(0.04-0.17)$ & $0.09(0.05-0.16)$ & $0.07(0.04-0.18)$ & 0.69 \\
\hline $\mathrm{HbA1c}(\%)$ & $6.12 \pm 0.92$ & $6.39 \pm 1.18$ & $6.07 \pm 0.85$ & 0.06 \\
\hline
\end{tabular}

Data are expressed as $\mathrm{n}(\%)$, mean \pm standard deviation, or median (interquartile range). BMI, body mass index; CABG, coronary artery bypass grafting; CKD, chronic kidney disease; CRP, C-reactive protein; DBP, diastolic blood pressure; DM, diabetes mellitus; HbA1c, hemoglobin A1c; HDL-C, high-density lipoprotein cholesterol; HT, hypertension; ID-TLR, ischemia driven target lesion revascularization; LDL-C, low-density lipoprotein cholesterol; MDA-LDL, malondialdehyde-modified low-density lipoprotein; MI, myocardial infarction; MVD, multi-vessel disease; PCI, percutaneous coronary intervention; SBP, systolic blood pressure; TGs, triglycerides. 


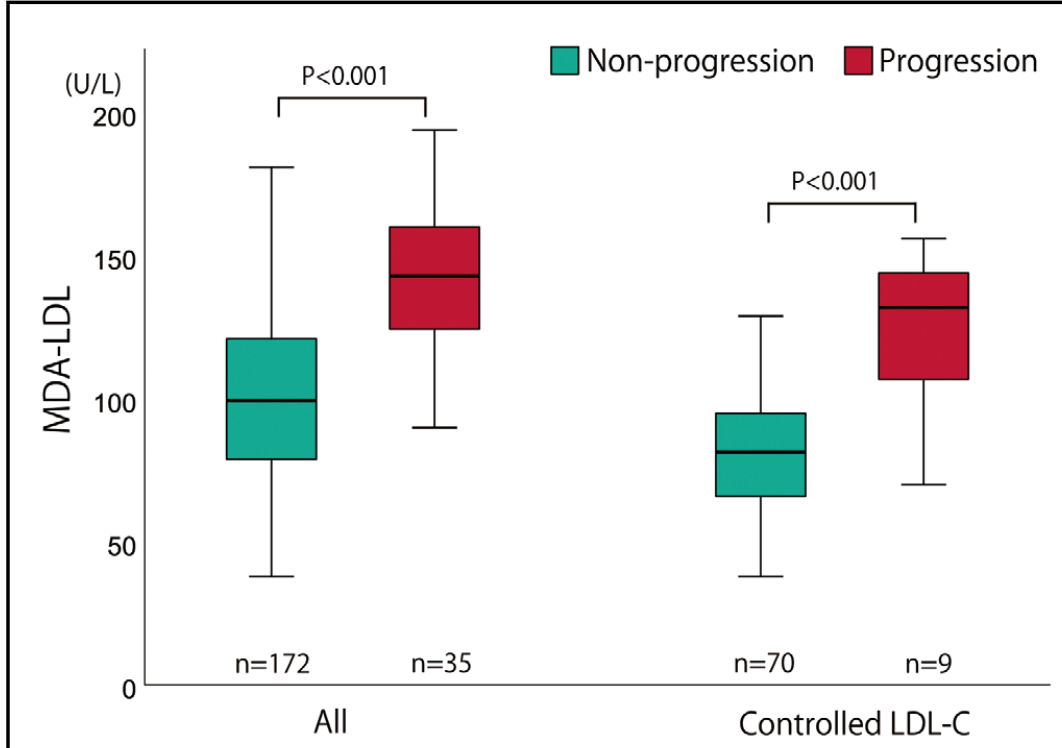

Figure 1. Relationship between angiographic progression and serum malondialdehyde-modified low-density lipoprotein (MDA-LDL) level. LDL-C, low-density lipoprotein cholesterol.

these, 213 patients who were treated with DES had followup coronary angiography (CAG) at 6-18 months after PCI and serial MDA-LDL measurement before PCI and at the time of follow-up CAG. After hemodialysis patients $(n=6)$ were excluded, 207 patients were retrospectively analyzed. The mean follow-up interval was $10.3 \pm 2.3$ months. The research protocol was approved by the Nagoya City University Graduate School of Medical Sciences and the Nagoya City University Hospital Institutional Review Board (Reference number: 60-19-0151).

The informed consent requirement was waived by the Board because we retrospectively accessed the database for analysis. All procedures performed in the present study involving human participants were in accordance with the ethical standards of the institutional research committee and with the Declaration of Helsinki.

\section{Blood Sampling and MDA-LDL Measurement}

Venous blood samples were collected after the patients fasted overnight. MDA-LDL levels were determined using an enzyme-linked immunosorbent assay kit (Sekisui Medical Co, Tokyo, Japan). ${ }^{15}$ After blood samples were collected, sera were separated within 6 h. Samples were diluted 2000fold in a dilution buffer containing $3.5 \mathrm{mmol} / \mathrm{L}$ sodium dodecyl sulfate (SDS). Duplicate $100 \mu \mathrm{L}$ portions of the samples were added to the wells of plates coated with a monoclonal antibody against MDA-LDL (ML25). MDAderived epitopes that may be buried in the lipid shell of LDL were expressed and recognized by ML25 in a protein assay with SDS-containing samples. The reaction was allowed to stand for $2 \mathrm{~h}$ at room temperature, then the plates were washed. Next, $\beta$-galactosidase-conjugated monoclonal antibody against apoB (AB16) was added. After incubation for $1 \mathrm{~h}$ at room temperature, o-nitrophenyl-galactopyranoside was added. After $2 \mathrm{~h}$, the reaction was stopped by adding $100 \mu \mathrm{L}$ of $0.2 \mathrm{~mol} / \mathrm{L}$ sodium carbonate $(\mathrm{pH} \mathrm{12})$. Absorbance at $415 \mathrm{~nm}$ was determined with a microplate reader. We tentatively defined $1 \mathrm{U} / \mathrm{L}$ MDA-LDL as the absorbance obtained with a primary standard concentration of $1 \mathrm{mg} / \mathrm{L}$. A calibration curve was prepared by diluting a reference serum as a secondary standard from 300 to 9,600-fold with dilution buffer and calculating the amount of MDA-LDL in the samples. High-density lipoprotein cholesterol (HDL-C), triglycerides (TGs), C-reactive protein (CRP), and hemoglobin Alc (HbAlc) were measured using standard laboratory procedures. LDL cholesterol (LDL-C) was measured using a direct homogenous assay (Kyowa Medex Co, Tokyo, Japan). ${ }^{\mathbf{1 6}}$

Blood samples were drawn just before PCI and at the time of follow-up CAG, and the patients were classified into the following 4 groups according to the serial levels of MDA-LDL, LDL-C, HDL-C, TGs, and CRP: persistent low, increased, attenuated, and persistent high respectively. High status was defined as a level more than the median value at baseline measurement, and persistent high was defined as the presence of high levels at both baseline and follow up. Patients with baseline high and follow-up low levels were considered the attenuated group, those with baseline low and follow-up high levels were considered the increased group, and those with low levels at both baseline and follow up were defined as the persistent low group.

\section{PCI Procedure and Clinical Follow up}

PCI was performed using standard techniques. The procedural strategy was left to the operator's discretion. DES was used as the first-line choice. Intravascular ultrasound or optical coherence tomography was routinely used to support appropriate stent implantation. All patients were pretreated with aspirin plus ticlopidine or clopidogrel before stenting, and at least 1 year of dual antiplatelet therapy was recommended. After PCI, lipid-lowering therapy, mainly with statin, was conducted with a target level of LDL-C $<100 \mathrm{mg} / \mathrm{dL}$, as recommended by the Japan Atherosclerosis Society Guidelines for the Diagnosis and Prevention of Atherosclerotic Cardiovascular Diseases (2012). ${ }^{17}$ Controlled LDL-C was defined as LDL-C $<100 \mathrm{mg} / \mathrm{dL}$ both before PCI and at follow up. Smoking cessation, healthy diet, maintaining a healthy weight, and physical activity according to the patient's exercise capacity were recommended for each patient. Antihypertensive and antidiabetic drugs were prescribed at the discretion of 

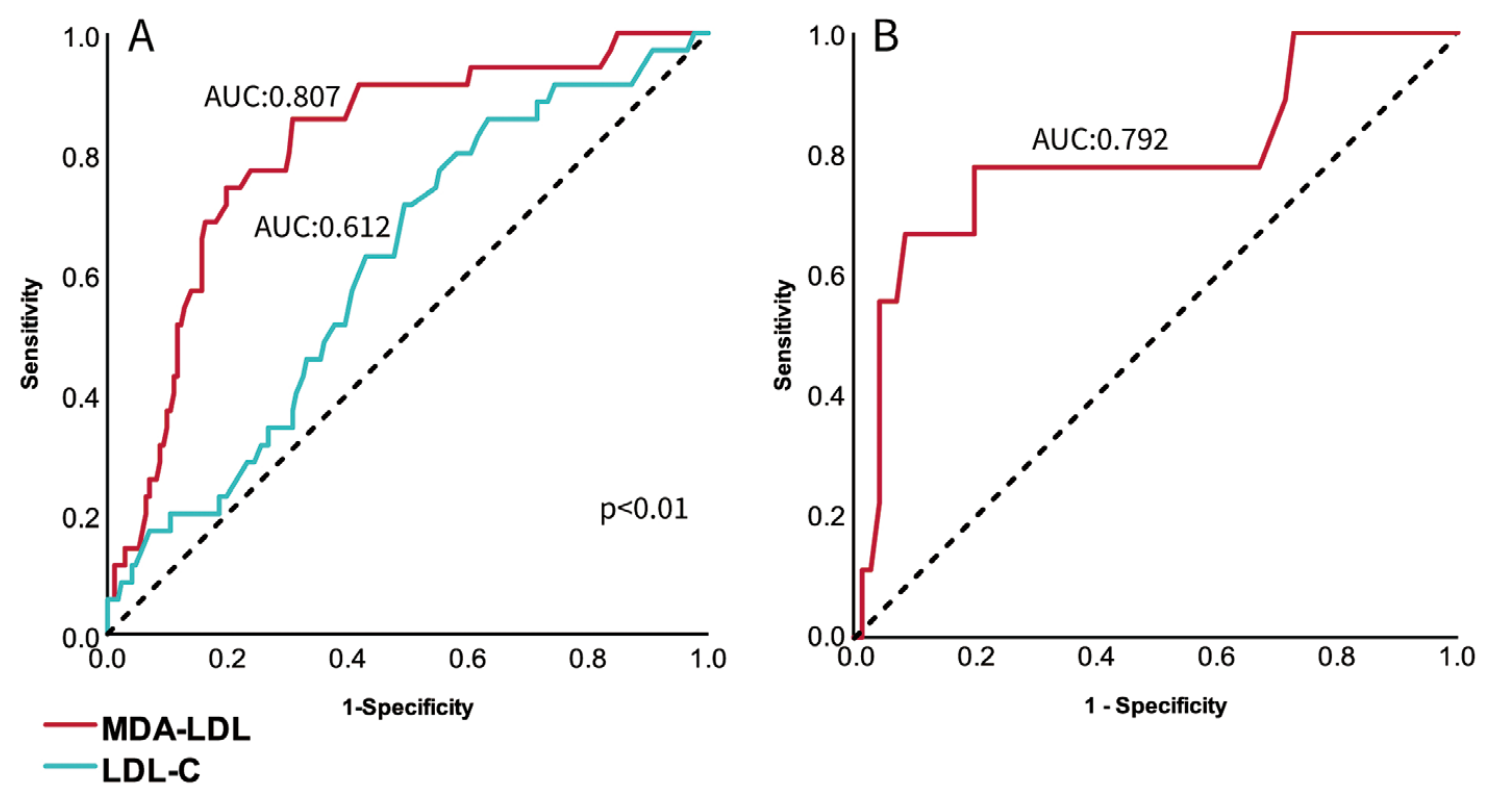

Figure 2. Receiver operating characteristic $(R O C)$ curve analysis. $R O C$ curve for predicting plaque progression at non-percutaneous coronary intervention (PCI) target lesions for malondialdehyde-modified low-density lipoprotein (MDA-LDL) and low-density lipoprotein cholesterol (LDL-C) before PCI $(\mathbf{A})$ and for MDA-LDL before PCl in patients with controlled LDL-C (B). AUC, area under the curve.
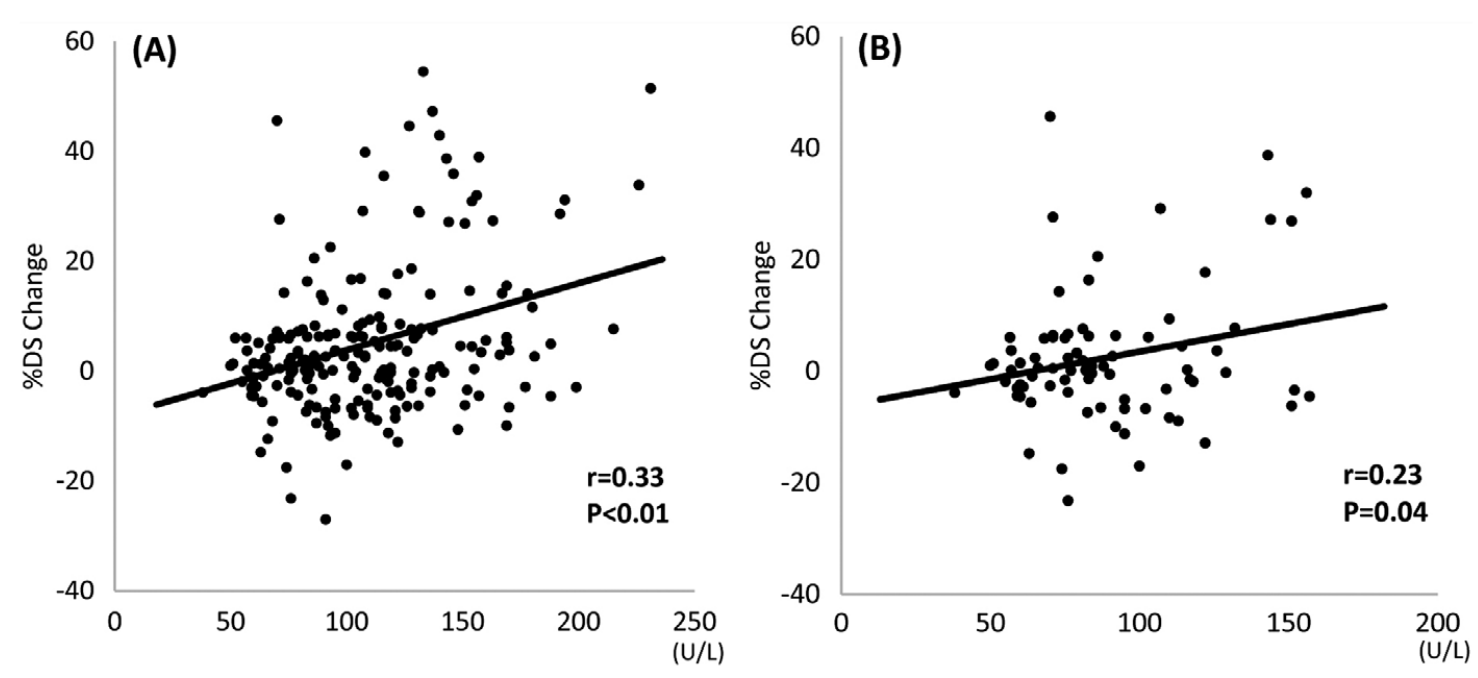

Figure 3. Correlation between the change in percent diameter stenosis (\%DS) and serum malondialdehyde-modified low-density lipoprotein (MDA-LDL) level before percutaneous coronary intervention (PCI) in all patients $(\mathbf{A})$ and in patients with controlled low-density lipoprotein cholesterol (B).

the attending physicians according to patient's age and other comorbidities. Follow-up CAG was performed for the onset of ischemic symptoms, objective signs of myocardial ischemia, or as a follow-up examination. Decision to perform and the timing of follow-up CAG were left to the discretion of the attending physician.

\section{Analysis of CAG}

CAG was performed with the standard Judkins technique, with intracoronary administration of $0.2 \mathrm{mg}$ nitroglycerin after intravenous administration of 3,000 U heparin. Offline quantitative CAG (QCA) was performed with an automated edge-detection program (Cardiovascular Measurement System; Medical Imaging Systems, Leiden, the Netherlands). The non-PCI target lesion was a de novo 


\begin{tabular}{|c|c|c|c|c|c|}
\hline \multirow[b]{2}{*}{ Variables } & \multicolumn{5}{|c|}{ MDA-LDL type } \\
\hline & $\begin{array}{l}\text { Persistent low } \\
(n=80)\end{array}$ & $\begin{array}{c}\text { Increased } \\
(n=23)\end{array}$ & $\begin{array}{c}\text { Attenuated } \\
(n=40)\end{array}$ & $\begin{array}{c}\text { Persistent high } \\
(n=64)\end{array}$ & $P$ value \\
\hline Age (years) & $71.4 \pm 8.4$ & $71.0 \pm 7.5$ & $74.0 \pm 6.3$ & $68.8 \pm 9.5$ & 0.022 \\
\hline Male & $58(72.5)$ & $15(65.2)$ & $29(72.5)$ & $46(71.9)$ & 0.92 \\
\hline BMI $\left(\mathrm{kg} / \mathrm{m}^{2}\right)$ & $24.1 \pm 3.9$ & $23.5 \pm 3.4$ & $24.0 \pm 2.7$ & $24.6 \pm 2.8$ & 0.51 \\
\hline HT & $75(93.8)$ & $18(78.3)$ & $35(87.5)$ & $56(87.5)$ & 0.19 \\
\hline DM & $39(48.8)$ & $12(52.2)$ & $10(25.0)$ & $28(43.8)$ & 0.067 \\
\hline Smoking & $20(25.0)$ & $5(21.7)$ & $12(30.0)$ & $17(26.6)$ & 0.90 \\
\hline \multicolumn{6}{|l|}{ LDL-C } \\
\hline At $\mathrm{PCl}$ & $87.7 \pm 26.1$ & $94.1 \pm 17.8$ & $115.3 \pm 28.7$ & $116.8 \pm 26.3$ & $<0.001$ \\
\hline At follow up & $83.6 \pm 21.9$ & $100.5 \pm 25.0$ & $84.5 \pm 18.7$ & $113.9 \pm 26.3$ & $<0.001$ \\
\hline \multicolumn{6}{|l|}{ HDL-C } \\
\hline At $\mathrm{PCl}$ & $49.3 \pm 15.2$ & $50.0 \pm 12.2$ & $47.1 \pm 13.5$ & $45.4 \pm 12.2$ & 0.30 \\
\hline At follow up & $51.6 \pm 14.2$ & $53.0 \pm 12.9$ & $48.8 \pm 12.5$ & $47.0 \pm 12.3$ & 0.12 \\
\hline \multicolumn{6}{|l|}{ TGs } \\
\hline At $\mathrm{PCl}$ & $130.2 \pm 59.4$ & $168.0 \pm 79.4$ & $153.7 \pm 67.6$ & $154.3 \pm 58.8$ & 0.026 \\
\hline At follow up & $136.2 \pm 66.1$ & $158.4 \pm 94.1$ & $151.4 \pm 69.1$ & $168.1 \pm 74.3$ & 0.073 \\
\hline \multicolumn{6}{|l|}{ CRP } \\
\hline At $\mathrm{PCl}$ & $0.09(0.05-0.15)$ & $0.08(0.06-0.14)$ & $0.12(0.05-0.18)$ & $0.13(0.05-0.25)$ & 0.64 \\
\hline At follow up & $0.08(0.04-0.15)$ & $0.07(0.05-0.17)$ & $0.09(0.04-0.29)$ & $0.07(0.04-0.16)$ & 0.61 \\
\hline \multicolumn{6}{|l|}{$\mathrm{HbA} 1 \mathrm{c}$} \\
\hline At $\mathrm{PCl}$ & $6.31 \pm 0.88$ & $6.37 \pm 0.88$ & $6.01 \pm 0.60$ & $6.59 \pm 1.16$ & 0.023 \\
\hline At follow up & $5.98 \pm 0.72$ & $6.27 \pm 1.13$ & $5.86 \pm 0.51$ & $6.41 \pm 1.16$ & 0.006 \\
\hline \multicolumn{6}{|l|}{$\mathrm{QCA}$ at $\mathrm{PCl}$} \\
\hline Reference diameter (mm) & $2.27 \pm 0.07$ & $2.23 \pm 0.15$ & $2.21 \pm 0.09$ & $2.24 \pm 0.08$ & 0.97 \\
\hline MLD (mm) & $1.39 \pm 0.06$ & $1.39 \pm 0.15$ & $1.40 \pm 0.10$ & $1.39 \pm 0.07$ & 1.00 \\
\hline$\% D S$ & $39.12 \pm 1.79$ & $38.32 \pm 3.39$ & $38.35 \pm 3.28$ & $39.35 \pm 2.10$ & 0.99 \\
\hline \multicolumn{6}{|l|}{ QCA at follow up } \\
\hline Reference diameter (mm) & $2.29 \pm 0.07$ & $2.21 \pm 0.16$ & $2.19 \pm 0.09$ & $2.25 \pm 0.09$ & 0.89 \\
\hline MLD (mm) & $1.37 \pm 0.06$ & $1.35 \pm 0.14$ & $1.26 \pm 0.12$ & $1.13 \pm 0.07$ & 0.14 \\
\hline$\% \mathrm{DS}$ & $40.19 \pm 2.02$ & $40.17 \pm 3.58$ & $43.56 \pm 4.06$ & $50.39 \pm 2.56$ & 0.02 \\
\hline Change in MLD (mm) & $-0.03 \pm 0.03$ & $-0.04 \pm 0.09$ & $-0.14 \pm 0.05$ & $-0.25 \pm 0.05$ & $<0.01$ \\
\hline Change in \%DS & $1.07 \pm 1.03$ & $1.86 \pm 2.39$ & $5.22 \pm 2.53$ & $11.04 \pm 1.88$ & $<0.01$ \\
\hline
\end{tabular}

Data are expressed as $n(\%)$, mean \pm standard deviation, median (interquartile range), or mean \pm standard error. QCA, quantitative coronary angiography; MLD, minimal lumen diameter; \%DS, percent diameter stenosis. Other abbreviations as in Table 1.

lesion that was not responsible for the ischemic symptoms or positive functional ischemic tests before PCI. All coronary sites that looked stenotic were visually screened at both baseline and follow up. When a patient had a single non-PCI target lesion, it was analyzed. When more than one measurable lesion was present, the lesion with the largest increase in percent diameter stenosis $(\% \mathrm{DS})$ between baseline and follow up was analyzed as the index lesion. In the case of a development of new stenosis in a normal segment at baseline, QCA at baseline was manually performed in the equal segment of new stenosis at follow-up angiography. To assess lesion progression, the same angiographic projections were analyzed at baseline and follow up, whereas coronary branches were used as markers to identify the coronary segments. As in a previous study, ${ }^{8}$ angiographic progression of a non-PCI target lesion was defined as any of the following based on QCA analysis: (1) $\geq 10 \%$ diameter reduction of a pre-existing stenosis $\geq 50 \%$; (2) $\geq 30 \%$ diameter reduction of a pre-existing stenosis $<50 \%$; (3) development of a new stenosis $\geq 30 \%$ diameter reduction in a segment that was normal at the time of PCI; and (4) progression of any lesion to total occlusion at follow-up CAG. We excluded lesions of grafted vessels and those within $20 \mathrm{~mm}$ of the anastomotic stenosis and the PCI target lesion. A stenosis $>50 \%$ was considered angiographically significant, and multivessel disease (MVD) was defined as $\geq 2$ vessels with angiographically significant stenosis. In-stent restenosis was defined as angiographic significant stenosis at the stented segment or its edges (5-mm segments adjacent to the stent). Coronary angiograms were reviewed separately by 2 independent observers.

\section{Statistical Analysis}

Continuous variables were compared using the Student's t-test, the Mann-Whitney U-test, and 1-way analysis of variance, as appropriate. The chi-squared test and Fisher's exact test were used to compare categorical variables. Receiver operating characteristic (ROC) curves were generated to assess the potential of MDA-LDL and LDL-C to predict angiographic progression of non-PCI target lesions. Comparison of areas under the curve (AUC) was performed with the chi-squared test. Correlations between serum MDA-LDL levels before PCI and changes in \%DS were estimated with Pearson's correlation coefficients. 


\begin{tabular}{|c|c|c|c|c|c|c|c|c|}
\hline \multirow{3}{*}{ Variables } & \multicolumn{2}{|c|}{ Univariate analysis } & \multicolumn{6}{|c|}{ Multivariate analysis } \\
\hline & \multirow[b]{2}{*}{$\begin{array}{c}\text { OR } \\
(95 \% \mathrm{Cl})\end{array}$} & \multirow[b]{2}{*}{$P$ value } & \multicolumn{2}{|c|}{ Model 1} & \multicolumn{2}{|c|}{ Model 2} & \multicolumn{2}{|c|}{ Model 3} \\
\hline & & & $\begin{array}{c}\text { OR } \\
(95 \% \mathrm{Cl})\end{array}$ & $P$ value & $\begin{array}{c}\text { OR } \\
(95 \% \mathrm{Cl})\end{array}$ & $P$ value & $\begin{array}{c}\text { OR } \\
(95 \% \mathrm{Cl})\end{array}$ & $P$ value \\
\hline Persistent high MDA-LDL & $\begin{array}{c}7.20 \\
(3.25-15.97)\end{array}$ & $<0.01$ & $\begin{array}{c}6.49 \\
(2.89-14.54)\end{array}$ & $<0.01$ & - & - & $\begin{array}{c}6.25 \\
(2.78-14.09)\end{array}$ & $<0.01$ \\
\hline Persistent low HDL-C & $\begin{array}{c}2.24 \\
(1.07-4.69)\end{array}$ & 0.03 & $\begin{array}{c}1.81 \\
(0.81-4.02)\end{array}$ & 0.15 & $\begin{array}{c}1.96 \\
(0.92-4.17)\end{array}$ & 0.08 & - & - \\
\hline Persistent high LDL-C & $\begin{array}{c}2.13 \\
(0.99-4.56)\end{array}$ & 0.05 & - & - & $\begin{array}{c}2.01 \\
(0.93-4.38)\end{array}$ & 0.08 & - & - \\
\hline Persistent high TGs & $\begin{array}{c}2.12 \\
(1.01-4.43)\end{array}$ & 0.05 & - & - & - & - & $\begin{array}{c}1.73 \\
(0.78-3.87)\end{array}$ & 0.18 \\
\hline MVD & $\begin{array}{c}1.99 \\
(0.95-4.15)\end{array}$ & 0.07 & $\begin{array}{c}1.48 \\
(0.67-3.29)\end{array}$ & 0.34 & $\begin{array}{c}1.80 \\
(0.85-3.83)\end{array}$ & 0.13 & $\begin{array}{c}1.65 \\
(0.74-3.66)\end{array}$ & 0.22 \\
\hline Age (years) & $\begin{array}{c}0.96 \\
(0.92-1.00)\end{array}$ & 0.07 & - & - & - & - & - & - \\
\hline Persistent high CRP & $\begin{array}{c}1.81 \\
(0.87-3.77)\end{array}$ & 0.11 & - & - & - & - & - & - \\
\hline Previous $\mathrm{PCl}$ & $\begin{array}{c}1.70 \\
(0.81-3.55)\end{array}$ & 0.16 & - & - & - & - & - & - \\
\hline CKD & $\begin{array}{c}0.58 \\
(0.25-1.36)\end{array}$ & 0.21 & - & - & - & - & - & - \\
\hline DM & $\begin{array}{c}1.31 \\
(0.63-2.72)\end{array}$ & 0.47 & - & - & - & - & - & - \\
\hline HT & $\begin{array}{c}1.40 \\
(0.39-5.01)\end{array}$ & 0.60 & - & - & - & - & - & - \\
\hline Smoking & $\begin{array}{c}0.98 \\
(0.43-2.24)\end{array}$ & 0.96 & - & - & - & - & - & - \\
\hline
\end{tabular}

$\mathrm{Cl}$, confidence interval; OR, odds ratio. Other abbreviations as in Table 1.

Logistic regression analysis was performed to identify potential predictors related to angiographic progression of non-PCI target lesions. Variables of patient characteristics with $P$ values $<0.10$ in the univariate model were used in the multivariate model. Covariates in univariate analysis for the prediction were persistent high MDA-LDL, persistent low HDL-C, persistent high LDL-C, persistent high TGs, MVD, age, persistent high CRP, previous PCI, chronic kidney disease, diabetes mellitus, hypertension, and smoking. The variables included in the multivariate analyses were as follows: (Model 1) persistent high MDALDL, persistent low HDL-C, and MVD; (Model 2) persistent low HDL-C, persistent high LDL-C, and MVD; and (Model 3) persistent high MDA-LDL, persistent high TGs, and MVD. A P value $<0.05$ was considered statistically significant. SPSS Statistics version 25 (SPSS Inc., Chicago, IL, USA) was used for all analyses.

\section{Results}

Patients' clinical, angiographic, and biochemical data are summarized in Table 1. Angiographic progression of the non-culprit lesion was observed in $35(16.9 \%)$ patients. Of these, 19 patients had $\geq 10 \%$ reduction in the diameter of a pre-existing $\geq 50 \%$ stenosis, 9 patients had $\geq 30 \%$ reduction in the diameter of a pre-existing $<50 \%$ stenosis, 2 patients had a new $\geq 30 \%$ stenosis in a previously normal lesion, and 5 patients had progression to total occlusion at follow-up angiography. Regarding the clinical and angiographic parameters, there were no significant differences between the 2 groups, with the exception that the rate of MVD and angiographic in-stent restenosis at follow up tended to be higher in the progression group. In the biochemical data, the progression group presented with significantly higher MDA-LDL and LDL-C levels compared with the nonprogression group before PCI and at follow-up measurements. TGs levels were higher and HDL-C levels were lower in the progression group at follow up. Figure 1 showed comparisons of MDA-LDL level before PCI between the progression and the non-progression group. Even in patients with controlled LDL-C, the progression group had a significantly higher MDA-LDL level than the non-progression group $(121.8 \pm 32.7 \mathrm{U} / \mathrm{L}$ vs. $84.9 \pm 24.9 \mathrm{U} / \mathrm{L}$, $\mathrm{P}<0.001)$. ROC curve analyses revealed that the difference in AUCs between MDA-LDL and LDL-C before PCI was statistically significant and that the MDA-LDL level before PCI could be a better predictor of angiographic progression than LDL-C level (Figure 2A). The MDALDL cut-off value before PCI for predicting angiographic progression was $126.5 \mathrm{U} / \mathrm{L}$ (sensitivity of 0.74 ; specificity of 0.80 ). Also, in the patients with controlled LDL-C, MDALDL before PCI could be a determinant of angiographic progression (cut-off value of $105 \mathrm{U} / \mathrm{L}$, sensitivity of 0.77 ; specificity of 0.80 ) (Figure 2B). There was positive correlation between \%DS changes and serum MDA-LDL levels before PCI not only in all patients but also in those with controlled LDL-C (Figure 3).

Patient characteristics and QCA analysis of non-PCI target lesions according to MDA-LDL type are shown in Table 2. Median values of MDA-LDL, LDL-C, HDL-C, TGs, and CRP before PCI were $107 \mathrm{U} / \mathrm{L}, 100 \mathrm{mg} / \mathrm{dL}$, $45 \mathrm{mg} / \mathrm{dL}, 133 \mathrm{mg} / \mathrm{dL}$, and $0.09 \mathrm{mg} / \mathrm{dL}$, respectively. In the persistent high MDA-LDL group, patients were younger. Furthermore, LDL-C and HbAlc were significantly higher 
compared to other groups. Although not statistically significant, TGs were higher and HDL-C was lower in the persistent high MDA-LDL group. The changes in minimal lesion diameter and \%DS were significantly different among the MDA-LDL groups. Univariate logistic regression analysis showed that persistent high MDA-LDL and persistent low HDL-C had significant association with angiographic progression of the non-PCI target lesion. Multivariate analysis revealed that persistent high MDALDL was an independent predictor regardless of the model (Table 3).

\section{Discussion}

To the best of our knowledge, this is the first report showing that MDA-LDL may be a potential marker of angiographic progression of non-PCI target coronary lesions in stable angina patients who were treated with DES and lipid-lowering therapy. Our main finding was that serum MDA-LDL level, in particular MDA-LDL before PCI, was strongly associated with angiographic progression. This association remained true even in the patients with controlled LDL-C. Furthermore, persistent high MDALDL was an independent predictor of angiographic progression.

\section{Disease Progression After PCI in the DES Era}

Since the appearance and development of DESs, the restenosis rate after PCI has markedly improved, resulting in a great reduction in the incidence of TLR.1,2 The development of current-generation DES, in conjunction with the advancement of intravascular imaging modalities to optimize stent deployment, has enabled safe and effective treatment, even in cases with complex lesions such as left main disease. ${ }^{18}$ In our study, all DESs were implanted under intravascular imaging guidance. Although angiographic in-stent restenosis, which may potentially reflect in-stent atherosclerotic progression (neoatherosclerosis), ${ }^{19}$ was relatively frequently observed in the progression group, the rate of TLR was acceptably low. In contrast, there is little evidence that PCI for stable CAD can reduce cardiac death or myocardial infarction compared with optimal medical therapy. ${ }^{20} \mathrm{PCI}$ is considered a "local treatment" for symptom relief and cannot prevent the development of new coronary atherosclerotic lesions at the non-stented segment that affects long-term prognosis after DES implantation. In line with a previous study showing that new coronary lesion development is common, ${ }^{21}$ this study demonstrated the high incidence of non-PCI target lesion progression, which has a potentially large effect on adverse cardiac events. ${ }^{6}$ Therefore, preventing new lesion progression has become an important therapeutic target to improve long-term clinical outcomes in CAD patients after DES implantation.

\section{Role of Oxidative Stress in CAD Progression}

Despite increased attention to the importance of CAD progression, the underlying mechanism is still not fully understood. The theory that oxidative stress triggers lipid oxidization and that oxidized LDL plays a crucial role in coronary atherogenesis has been suggested. ${ }^{22}$ Uptake of oxidized LDL leads to notable cholesterol deposition, converting macrophages to foam cells and promoting the development of atherosclerotic plaques. ${ }^{10}$ In addition, oxidized LDL induces various inflammatory activities includ- ing recruitment of monocytes, activation of lymphocytes, and secretion of cytokines and growth factors with accelerated smooth muscle cell proliferation and necrotic core formation. ${ }^{23}$ Although it is difficult to quantify intracoronary oxidative stress in daily clinical practice, we focused on MDA-LDL, a potential alternative marker to oxidative stress, to investigate the relationship with coronary plaque progression at non-stented sites. We showed that increased MDA-LDL was significantly associated with coronary plaque progression, supporting the hypothesis that exposure to oxidative stress may be associated with coronary atherosclerotic plaque progression.

Residual Risk After Optimal Secondary Prevention Therapy Based on accumulating evidence from lipid-lowering pharmacological therapy for the prevention of plaque progression $^{\mathbf{2 4}}$ and cardiovascular events, ${ }^{3}$ current guidelines strongly recommend statin use for CAD patients as a secondary prevention. ${ }^{25}$ However, a certain proportion of patients undergoing lipid-lowering therapy experience plaque progression, despite having well-controlled LDLC. ${ }^{26}$ LDL particles are heterogeneous in terms of composition both in size and density. ${ }^{27}$ It has been reported that small dense LDL (sdLDL) is associated with increased risk for cardiovascular disease ${ }^{28}$ because of easy absorption into the arterial wall, lower binding affinity for the LDL receptor, a prolonged plasma half-time and greater susceptibility to oxidation. ${ }^{29}$ The concentration of $\operatorname{sdLDL}$ is increased in individuals with high TGs, dysglycemia and the metabolic syndrome. ${ }^{30}$ In our study, high TGs and HbA1c, and low HDL-C were observed in patients with a high level of MDA-LDL. It may imply that potentially high sdLDL levels in patients with high MDA-LDL could lead to coronary atherosclerotic progression. One prior study referred to the relevance of hypertension and diabetes mellitus to plaque progression, ${ }^{\mathbf{2 6}}$ whereas another indicated residual inflammatory risk ${ }^{\mathbf{3 1}}$ in patients undergoing lipid-lowering therapy. These data suggest that plaque progression is a multifactorial phenomenon and decreasing the LDL-C level alone may be insufficient to prevent it. Therefore, it is important to comprehensively manage other risk factors such as smoking, body weight, blood pressure, TGs, diabetes, and metabolic syndrome by implementing healthy behaviors and pharmacotherapy. In our study population, most patients who exhibited plaque progression had been managed with standard treatment, with lifestyle education and medication including statin therapy. Still, little is known about the residual risk under adequate secondary prevention after DES-PCI. Useful markers to identify high-residual-risk populations and practical methods to intervene should be established.

\section{Clinical Implication}

The major clinical implication of this study is that MDALDL at PCI and a sustained increase in MDA-LDL may be useful markers for risk stratification in patients after DES-PCI. The present study found that the MDA-LDL level before PCI was higher in patients with angiographic progression and correlated to angiographic $\% \mathrm{DS}$ change, and that these findings are in line with a previous report that found that the pre-PCI MDA-LDL level is a predictor of cardiovascular events. ${ }^{14}$ In addition, the MDA-LDL level before PCI was significantly associated with coronary plaque progression, even under LDL-C control. By assessing MDA-LDL before PCI, high-risk patients may be 
identified in advance. Furthermore, in this study, serial assessment of MDA-LDL demonstrated that persistent high MDA-LDL could have an effect on angiographic progression. By measuring MDA-LDL not only before PCI but also during follow up, high-risk patients could be more accurately identified. Although our findings are hypothesis-generating and larger prospective studies are required to confirm the effectiveness of MDL-LDL-lowering therapy and MDA-LDL-guided pharmacotherapy, high MDA-LDL may be a target to treat in patients after DESPCI.

\section{Study Limitations}

This study had several limitations. First, it was a retrospective observational study with a relatively low number of Japanese patients recruited at a single center. In addition, indication of follow-up CAG was decided by the attending physician without strict criteria, and patients being treated with hemodialysis were excluded from the analysis because follow-up CAG in dialysis patients was performed only in cases with strongly suspected CAD recurrence and/or progression. Therefore, the population was highly biased. Further prospective studies are needed with larger populations. Second, lipid-lowering therapy was administered according to a 2012 Japanese guideline, and the target LDL-C level differed from that of current standard guidelines. Third, the relationship between MDA-LDL and LDL particle size is not clarified. More detailed evaluation of the lipid profile such as apolipoprotein B and sdLDL is required. Fourth, CAG is only a lumenogram and we did not incorporate intravascular imaging analysis in this study, so data on plaque burden and/or vulnerability were lacking. Fifth, although frequent in-stent restenosis in the progression group may suggest that MDA-LDL could be also related to in-stent neoatherosclerosis, it should be confirmed by further intravascular imaging and histopathological studies. Finally, angiographic progression is a surrogate marker for cardiovascular events. Clinical outcome data with longer follow up are needed.

\section{Conclusions}

Increased serum MDA-LDL was associated with angiographic plaque progression after DES implantation.

\section{Disclosures}

All authors received no support from sponsors and have no conflicts of interest to declare. N.O. is a member of Circulation Journal' Editorial Team.

\section{Data Availability}

The deidentified participant data will not be shared.

\section{References}

1. Piccolo R, Bonaa KH, Efthimiou O, Varenne O, Baldo A, Urban $\mathrm{P}$, et al. Drug-eluting or bare-metal stents for percutaneous coronary intervention: A systematic review and individual patient data meta-analysis of randomised clinical trials. Lancet 2019; 393: $2503-2510$.

2. Bønaa KH, Mannsverk J, Wiseth R, Aaberge L, Myreng Y, Nygård $\mathrm{O}$, et al. Drug-eluting or bare-metal stents for coronary artery disease. $N$ Engl J Med 2016; 375: 1242-1252.

3. Taguchi I, Iimuro S, Iwata H, Takashima H, Abe M, Amiya E, et al. High-dose versus low-dose pitavastatin in Japanese patients with stable coronary artery disease (REAL-CAD) a randomized superiority trial. Circulation 2018; 137: 1997-2009.

4. Cannon CP, Blazing MA, Giugliano RP, McCagg A, White JA,
Theroux P, et al. Ezetimibe added to statin therapy after acute coronary syndromes. N Engl J Med 2015; 372: 2387-2397.

5. Stone GW, Maehara A, Lansky AJ, de Bruyne B, Cristea E, Mintz GS, et al. A prospective natural-history study of coronary atherosclerosis. $N$ Engl J Med 2011; 364: 226-235.

6. Waters D, Craven TE, Lesperance J. Prognostic significance of progression of coronary atherosclerosis. Circulation 1993; 87: $1067-1075$

7. Kaski JC, Chester MR, Chen L, Katritsis D. Rapid angiographic progression of coronary artery disease in patients with angina pectoris. Circulation 1995; 92: 2058-2065.

8. Zouridakis E, Avanzas P, Arroyo-Espliguero R, Fredericks S, Kaski JC. Markers of inflammation and rapid coronary artery disease progression in patients with stable angina pectoris. Circulation 2004; 110: 1747-1753.

9. Puri R, Ballantyne CM, Hoogeveen RC, Shao M, Barter P, Libby $\mathrm{P}$, et al. Lipoprotein(a) and coronary atheroma progression rates during long-term high-intensity statin therapy: Insights from SATURN. Atherosclerosis 2017; 263: 137-144.

10. Borén J, Chapman MJ, Krauss RM, Packard CJ, Bentzon JF, Binder CJ, et al. Low-density lipoproteins cause atherosclerotic cardiovascular disease: Pathophysiological, genetic, and therapeutic insights: A consensus statement from the European Atherosclerosis Society Consensus Panel. Eur Heart J 2020; 41: $2313-2330$.

11. Amaki T, Suzuki T, Nakamura F, Hayashi D, Imai Y, Morita $\mathrm{H}$, et al. Circulating malondialdehyde modified LDL is a biochemical risk marker for coronary artery disease. Heart 2004; 90: $1211-1213$.

12. Matsuo Y, Kubo T, Okumoto Y, Ishibashi K, Komukai K, Tanimoto T, et al. Circulating malondialdehyde-modified lowdensity lipoprotein levels are associated with the presence of thin-cap fibroatheromas determined by optical coherence tomography in coronary artery disease. Eur Heart J Cardiovasc Imaging 2013; 14: 43-50.

13. Holvoet $\mathrm{P}$, Collen $\mathrm{D}$, Van De Werf F. Malondialdehyde-modified LDL as a marker of acute coronary syndromes. J Am Med Assoc 1999; 281: $1718-1721$.

14. Ito T, Fujita H, Tani T, Ohte N. Malondialdehyde-modified low-density lipoprotein is a predictor of cardiac events in patients with stable angina on lipid-lowering therapy after percutaneous coronary intervention using drug-eluting stent. Atherosclerosis 2015; 239: 311-317.

15. Kotani K, Maekawa M, Kanno T, Kondo A, Toda N, Manabe M. Distribution of immunoreactive malondialdehyde-modified low-density lipoprotein in human serum. Biochim Biophys Acta (BBA)/Lipids Lipid Metab 1994; 1215: 121-125.

16. Sugiuchi H, Irie T, Uji Y, Ueno T, Chaen T, Uekama K, et al. Homogeneous assay for measuring low-density lipoprotein cholesterol in serum with triblock copolymer and $\alpha$-cyclodextrin sulfate. Clin Chem 1998; 44: 522-531.

17. Teramoto T, Sasaki J, Ishibashi S, Birou S, Daida H, Dohi S, et al. Diagnosis of atherosclerosis: Executive summary of the Japan Atherosclerosis Society (JAS) guidelines for the diagnosis and prevention of atherosclerotic cardiovascular diseases in Japan 2012 version. J Atheroscler Thromb 2014; 21: 296-298.

18. Stone GW, Pieter Kappetein A, Sabik JF, Pocock SJ, Morice MC, Puskas J, et al. Five-year outcomes after PCI or CABG for left main coronary disease. $N$ Engl J Med 2019; 381: 1820-1830.

19. Nakazawa G, Otsuka F, Nakano M, Vorpahl M, Yazdani SK, Ladich E, et al. The pathology of neoatherosclerosis in human coronary implants bare-metal and drug-eluting stents. $\mathrm{J}$ Am Coll Cardiol 2011; 57: 1314-1322.

20. Boden WE, O'Rourke RA, Teo KK, Hartigan PM, Maron DJ, Kostuk WJ, et al. Optimal medical therapy with or without PCI for stable coronary disease. N Engl J Med 2007; 356: 1503-1516.

21. Lautamäki A, Airaksinen KEJ, Kiviniemi T, Vinco G, Ribichini F, Gunn J, et al. Prognosis and disease progression in patients under 50 years old undergoing PCI: The CRAGS (Coronary aRtery diseAse in younG adultS) study. Atherosclerosis 2014; 235: $483-487$.

22. Madamanchi NR, Vendrov A, Runge MS. Oxidative stress and vascular disease. Arterioscler Thromb Vasc Biol 2005; 25: 29-38.

23. Ley K, Miller YI, Hedrick CC. Monocyte and macrophage dynamics during atherogenesis. Arterioscler Thromb Vasc Biol 2011; 31: 1506-1516.

24. Tsujita K, Sugiyama S, Sumida H, Shimomura H, Yamashita T, Yamanaga $\mathrm{K}$, et al. Impact of dual lipid-lowering strategy with ezetimibe and atorvastatin on coronary plaque regression in patients with percutaneous coronary intervention: The 
Multicenter Randomized Controlled PRECISE-IVUS Trial. $J$ Am Coll Cardiol 2015; 66: 495-507.

25. Knuuti J, Wijns W, Saraste A, Capodanno D, Barbato E, FunckBrentano C, et al. 2019 ESC Guidelines for the diagnosis and management of chronic coronary syndromes. Eur Heart J 2020; 41: $407-477$.

26. Bayturan O, Kapadia S, Nicholls SJ, Tuzcu EM, Shao M, Uno $\mathrm{K}$, et al. Clinical predictors of plaque progression despite very low levels of low-density lipoprotein cholesterol. $\mathrm{J} \mathrm{Am} \mathrm{Coll} \mathrm{Car-}$ diol 2010; 55: 2736-2742.

27. Krauss RM, Burke DJ. Identification of multiple subclasses of plasma low density lipoproteins in normal humans. $J$ Lipid Res 1982; 23: 97-104.

28. Hoogeveen RC, Gaubatz JW, Sun W, Dodge RC, Crosby JR, Jiang J, et al. Small dense low-density lipoprotein-cholesterol concentrations predict risk for coronary heart disease: The Ath- erosclerosis Risk In Communities (ARIC) study. Arterioscler Thromb Vasc Biol 2014; 34: 1069-1077.

29. Mikhailidis DP, Elisaf M, Rizzo M, Berneis K, Griffin B, Zambon A, et al. "European panel on low density lipoprotein (LDL) subclasses": A statement on the pathophysiology, atherogenicity and clinical significance of LDL subclasses. Curr Vasc Pharmacol 2011; 9: 533-571.

30. Gazi I, Tsimihodimos V, Filippatos T, Bairaktari E, Tselepis $\mathrm{AD}$, Elisaf M. Concentration and relative distribution of lowdensity lipoprotein subfractions in patients with metabolic syndrome defined according to the National Cholesterol Education Program criteria. Metabolism 2006; 55: 885-891.

31. Guedeney P, Claessen BE, Kalkman DN, Aquino M, Sorrentino $\mathrm{S}$, Giustino $\mathrm{G}$, et al. Residual inflammatory risk in patients with low LDL cholesterol levels undergoing percutaneous coronary intervention. J Am Coll Cardiol 2019; 73: 2401-2409. 